\title{
Bio-functionalization of Monodisperse Magnetic Nanoparticles and Their Use as Biomolecular Labels in a Magnetic Tunnel Junction Based Sensor
}

\author{
Stephanie G. Grancharov, ${ }^{\dagger,}$ Hao Zeng, ${ }^{\dagger, l}$ Shouheng Sun, ${ }^{\dagger}$ Shan X. Wang, ${ }^{\S}$ Stephen O’Brien, \\ C. B. Murray, ${ }^{\dagger}$ J. R. Kirtley, ${ }^{\dagger}$ and G. A. Held ${ }^{*} \dagger$ \\ IBM T.J. Watson Research Center, P.O. Box 218, Yorktown Heights, New York 10598, Department of Applied \\ Physics \& Applied Mathematics, Columbia University, New York, New York 10027, and Department of \\ Materials Science and Engineering, Stanford University, Stanford, California 94305
}

Received: March 3, 2005; In Final Form: April 25, 2005

\begin{abstract}
Monodisperse magnetic nanoparticles (NPs) could enable the ultra-sensitive magnetic detection of biological analytes. However, rendering these particles biocompatible has remained a challenge. We report the biofunctionalization and detection of 12-nm manganese ferrite NPs. We have achieved the site-specific binding of biotin-functionalized NPs onto avidin-patterned silicon oxide substrates and DNA-functionalized NPs onto complementary DNA-patterned silicon oxide substrates. Utilizing scanning SQUID microscopy, we show that these substrate-bound NPs retain their magnetic properties. Finally, we demonstrate a novel method of detecting either protein binding or DNA hybridization at room temperature using the NPs and a magnetic tunnel-junction-based biosensor situated in orthogonal magnetic fields.
\end{abstract}

\section{Introduction}

The bio-functionalization of monodisperse magnetic nanoparticles (NPs) of size $\sim 10-20 \mathrm{~nm}$ is of great interest as it would enable the ultra-sensitive magnetic detection of both proteins and nucleic acids. ${ }^{1}$ Given their extremely small size and high magnetization, such nanoparticles could also be used to bind and transport proteins, nucleic acids, and other biomolecules through microfluidic networks and, following introduction into a living organism, ${ }^{2}$ they could provide a means of monitoring and influencing cellular processes. While methods of synthesizing monodisperse and magnetically stable ferrite NPs with size smaller than $20 \mathrm{~nm}$ have been developed, ${ }^{3,4}$ the encapsulation of these particles within an overlayer of biologically active molecules and their subsequent stabilization in a physiological medium has remained a challenge. In this paper, we present the bio-functionalization and detection of 12-nm manganese ferrite $\left(\mathrm{MnFe}_{2} \mathrm{O}_{4}\right)$ NPs. We have achieved the sitespecific binding of biotin-functionalized magnetic NPs onto avidin-patterned silicon oxide substrates and DNA-functionalized magnetic NPs onto complementary DNA-patterned silicon oxide substrates. Scanning SQUID microscopy images of these substrate-bound NPs show that they retain their magnetic properties and thus can be detected by a magnetic-tunneljunction $^{5}$ (MTJ) field sensor. Finally, we demonstrate a novel method of detecting either protein binding or DNA hybridization at room temperature using the NPs and a MTJ biosensor situated in orthogonal magnetic fields.

The magnetic detection of biological analytes is potentially more sensitive than optical detection. ${ }^{6-8}$ Additionally, it avoids

* Corresponding author. Phone: 914-945-2609. E-mail: gaheld@ us.ibm.com

IBM T.J. Watson Research Center.

$\doteqdot$ Columbia University

$\S$ Stanford University.

" Present address: Department of Physics, State University of New York, Buffalo, NY. the effects of NP overlayer resistivity which is associated with conductivity-based detection..$^{9,10}$ The detection of magnetic NPs of size 10-20 nm also provides advantages over detection of micrometer-sized latex particles embedded with magnetic media: 6-8 The nanoscopic size of the particles allows each to be bound by a single analyte, while their strong magnetization should allow detection of a single particle. ${ }^{1}$ Further, between 1 and $10^{4}$ particles could bind and be detected at a $1-\mu \mathrm{m}^{2}$ magnetic field sensor, resulting in a dynamic range spanning 4 orders of magnitude. The detection scheme reported here does not require modulated ac magnetic fields. ${ }^{6,7}$ This fact, combined with the micrometer size of the tunnel junction, suggests that this detection method could potentially be scaled into a multianalyte "lab-on-a-chip" detector.

\section{Materials and Methods}

Synthesis of 12-nm $\mathrm{MnFe}_{2} \mathrm{O}_{4}$ Nanoparticles. Amounts of $2 \mathrm{mmol}$ of $\mathrm{Fe}(\mathrm{acac})_{3}, 1 \mathrm{mmol}$ of $\mathrm{Mn}(\mathrm{acac})_{2}, 10 \mathrm{mmol}$ of 1,2-hexadecandiol, $6 \mathrm{mmol}$ of oleic acid, $6 \mathrm{mmol}$ of oleylamine, and $20 \mathrm{~mL}$ of benzyl ether were heated at $110^{\circ} \mathrm{C}$ for $1 \mathrm{~h}$, with $\mathrm{N}_{2}$ flow. The temperature was then raised to $210{ }^{\circ} \mathrm{C}$, with a heating rate of about $8^{\circ} \mathrm{C} / \mathrm{min}$ and kept at that temperature for $2 \mathrm{~h}$. Under $\mathrm{N}_{2}$ blanketing, the mixture was heated to the reflux temperature of about $295{ }^{\circ} \mathrm{C}$, and kept refluxing for $1 \mathrm{~h}$. The solution mixture was cooled to room temperature, and the NPs were precipitated out by adding ethanol. A NdFeB permanent magnet was used to separate particles from the brown supernatant. The product, $12-\mathrm{nm}$ cubelike $\mathrm{MnFe}_{2} \mathrm{O}_{4} \mathrm{NPs}$, was washed with ethanol and redissolved in hexane to form a stable dispersion with a concentration of $\sim 5 \mathrm{mg} / \mathrm{mL}$.

Preparation of Biotinylated $\mathrm{MnFe}_{2} \mathrm{O}_{4}$ Nanoparticles. $\mathrm{MnFe}_{2} \mathrm{O}_{4}$ NPs were precipitated from $0.2 \mathrm{~mL}$ of the hexane suspension described above with methanol and dried under vacuum. The NPs were then re-suspended in chloroform with $5.5 \times 10^{-6}$ moles of the phospholipid 1,2-distearoyl-sn-glycero3-phosphoethanolamine- $N$-[biotinyl(poly(ethylene glycol))2000] 

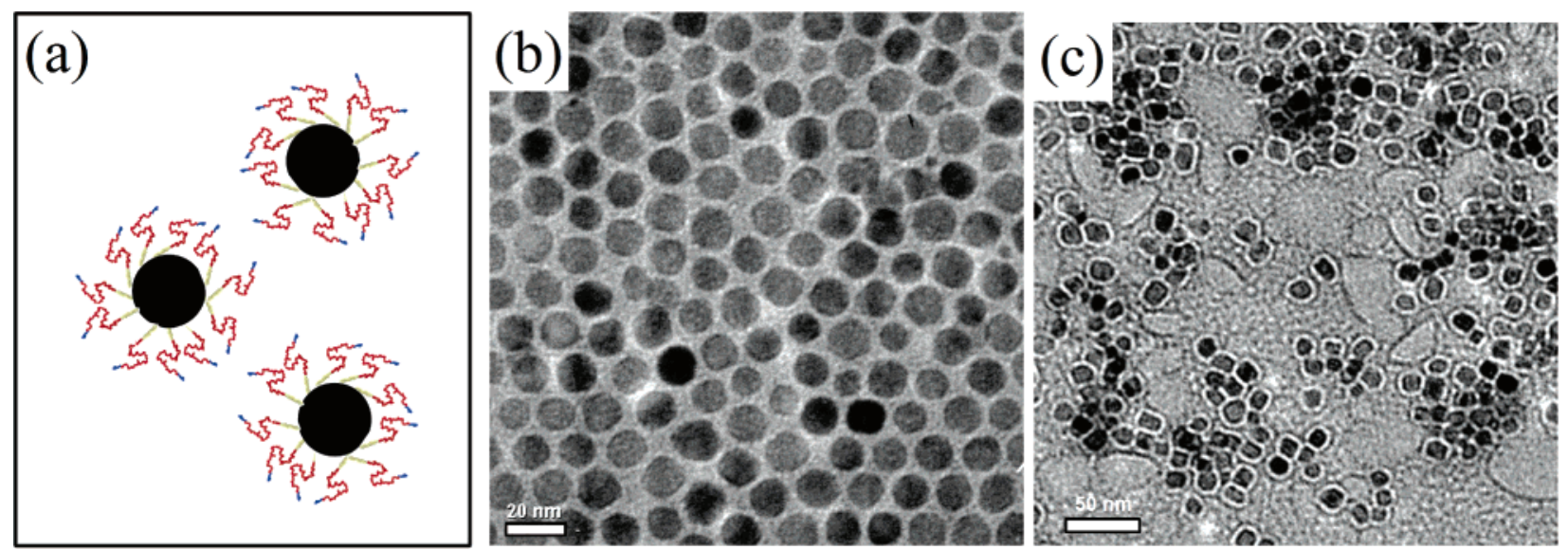

Figure 1. (a) Schematic of magnetic NPs dispersed in water. The ligands conjugated to the particles are comprised of a hydrophobic distearoyl phospholipid (yellow), hydrophilic poly(ethylene glycol) (red), and a biofunctional end group (blue) of either biotin or maleimide (for attachment of DNA). (b) TEM micrograph of $\mathrm{MnFe}_{2} \mathrm{O}_{4}$ NPs deposited from dispersion in hexane. (c) TEM micrograph of biotinPEG(2000)-PE-functionalized NPs deposited from dispersion in water. Sample is negatively stained with $1 \%$ phosphotungstic acid so that the phospholipids surrounding each NP appear as a white ring.

(biotinPEG(2000)-PE) or, alternately, an equal amount of either 1,2-distearoyl-sn-glycero-3-phosphoethanolamine- $N$-[methoxy(poly(ethylene glycol))-2000] (mPEG(2000)-PE) or 1,2-distearoyl-sn-glycero-3-phosphoethanolamine- $N$-[maleimide(poly(ethylene glycol))2000] (maleimidePEG(2000)-PE) (Avanti Polar Lipids). After shaking for $1 \mathrm{~h}$ and evaporating the chloroform, $0.2 \mathrm{~mL}$ of phosphate-buffered saline (PBS) solution was added to the dried particles, and the solution was heated to $80^{\circ} \mathrm{C}$ for $12 \mathrm{~h}$ to create a clear suspension of biotinPEG(2000)PE-conjugated NPs. To purify the solution and remove any empty micelles that may have formed from the biotinPEG(2000)-PE, the solution was centrifuged at $22000 \mathrm{~g}$ for $30 \mathrm{~min}$. The supernantent was then discarded, and the precipitant was resuspended in $0.5 \mathrm{~mL}$ of PBS solution.

Preparation of DNA-Functionalized $\mathrm{MnFe}_{2} \mathrm{O}_{4}$ Nanoparticles. To covalently bind single-stranded DNA (ssDNA) to NPs conjugated with the ligand maleimidePEG(2000)-PE, the particles were incubated with $5 \mathrm{nmol}$ of $5^{\prime}$ (ThiolC6)TTACTCGAGGGATCCTAGTC-3' (Midland Certified Reagent Company) dissolved in $100 \mu \mathrm{L}$ of PBS solution for $12 \mathrm{~h}$. This solution was then centrifuged at $22000 \mathrm{~g}$ for $30 \mathrm{~min}$. The supernatent was then discarded, and the precipitant was resuspended in $0.5 \mathrm{~mL}$ of PBS solution.

Stamp Mold Production. PDMS stamp molds were produced under common photolithographic conditions using NR8-9000 negative resist spun on silicon wafers for $120 \mathrm{~s}$ at $1500 \mathrm{rpm}$ to produce a thickness of $10 \mu \mathrm{m}$. After baking for $10 \mathrm{~min}$ at $70{ }^{\circ} \mathrm{C}$, then ramping up to $100{ }^{\circ} \mathrm{C}$ for an additional 3 min of bake time, the wafer was exposed through a photomask for $60 \mathrm{~s}$ using a Karl Suss contact printer operating at $9 \mathrm{~mW} /$ $\mathrm{cm}^{2}$. The photomasks consisted of line width features ranging from 2 to $200 \mu \mathrm{m}$ with $2-20 \mu \mathrm{m}$ in spacing between the lines. The resist-coated wafers were then placed into an oven at $90{ }^{\circ} \mathrm{C}$, ramped to $100{ }^{\circ} \mathrm{C}$, and postexposure-baked at $100{ }^{\circ} \mathrm{C}$ for $4 \mathrm{~min}$. The wafers were finally developed for $55 \mathrm{~s}$ using RD-6 developer solution, rinsed well with water for $1 \mathrm{~min}$, and nitrogen-dried.

Preparation of PDMS Stamp. The PDMS rubber stamps used for microfluidic network protein patterning onto $\mathrm{SiO}_{2}$ were prepared by mixing for 2 min a 10:1 ratio (v/w) of elastomer to hardener (Sylgard 184, Dow Corning) and pouring it over the stamp mold. After allowing the stamp to degas at room temperature for $1 \mathrm{~h}$, the stamp was cured for $1.5 \mathrm{~h}$ at $60{ }^{\circ} \mathrm{C}$ to cross-link the polymer. It was then removed from the substrate and oxidized in an rf-induced $\mathrm{O}_{2}$ plasma for $5 \mathrm{~min}$ to hydrophilize the surface. Stamps were then cleaned and stored in ethanol.

Patterning Avidin on $\mathrm{SiO}_{2}$ Surface. The above PDMS stamp was removed from the ethanol solution, dried with a stream of $\mathrm{N}_{2}$ gas, and subsequently incubated in $10 \%$ bovine serum albumin (BSA) solution for $20 \mathrm{~min}$. The stamp was then rinsed with DI water and again dried with $\mathrm{N}_{2}$ gas. Clean $\mathrm{SiO}_{2}$ substrates were silanized by incubation for $15 \mathrm{~min}$ in $0.02 \mathrm{~mL}$ of (aminopropyl)triethoxysilane (APTES) in $50 \mathrm{~mL}$ of 2-propanol. Stamps were then placed on the substrate, and contact between the two materials was achieved by gentle manual pressure to seal the channel walls. To $4 \mathrm{~mL}$ of a buffered aqueous solution of FITC-labeled avidin (Sigma, A-2050) were added $0.5 \mathrm{mg}$ each of NHSS ( $n$-hydroxysulfosuccinimide) and EDC ( $N$-ethyl$N^{\prime}$ (3-dimethylainopropyl)-carbodiimide), which improve the coupling efficiency between the avidin and the amines on the $\mathrm{SiO}_{2}$ substrate. ${ }^{11}$ Drops of this solution were then placed at one end of the microchannels, through which the solution flowed by capillary action. At the other end of the stamp, excess solution was absorbed by placement of blotting paper. To remove unbound protein from the substrate, the channels were flushed with $1 \%$ of the blocking agent BSA in PBS solution. After removing the stamp from the substrate while both are immersed in a $1 \%$ BSA solution, the substrate surface was blocked for nonspecific binding by incubating further in this solution for 10 min, then rinsed with DI water, and dried with $\mathrm{N}_{2}$ gas.

Patterning DNA on $\mathrm{SiO}_{2}$ Surface. Ten nanomoles of 5'(biotin)GACTAGGATCCCTCGAGTAA-3' (Midland Certified Reagent Company) were dissolved in $500 \mu \mathrm{L}$ of PBS solution. $\mathrm{A} \mathrm{SiO}_{2}$ substrate patterned with avidin was immersed in the solution and allowed to incubate for $12 \mathrm{~h}$.

Sample Preparation and Characterization. Samples studied with transmission electron microscopy (TEM) were prepared by placing one drop of a NP dispersion (hexane or aqueous based) onto the amorphous carbon substrate of a TEM grid and then drying the grid under vacuum at $100{ }^{\circ} \mathrm{C}$ for several hours. Images with phospholipids (Figure 1c) were additionally stained with one drop of a $1 \%$ phosphotungstic acid and dried under vacuum for $1 \mathrm{~h}$. TEM images were taken with a Philips CM-12 $120 \mathrm{keV}$ TEM.

Samples studied with X-ray diffraction (XRD) were prepared by drying the NP dispersion onto a glass substrate under vacuum 

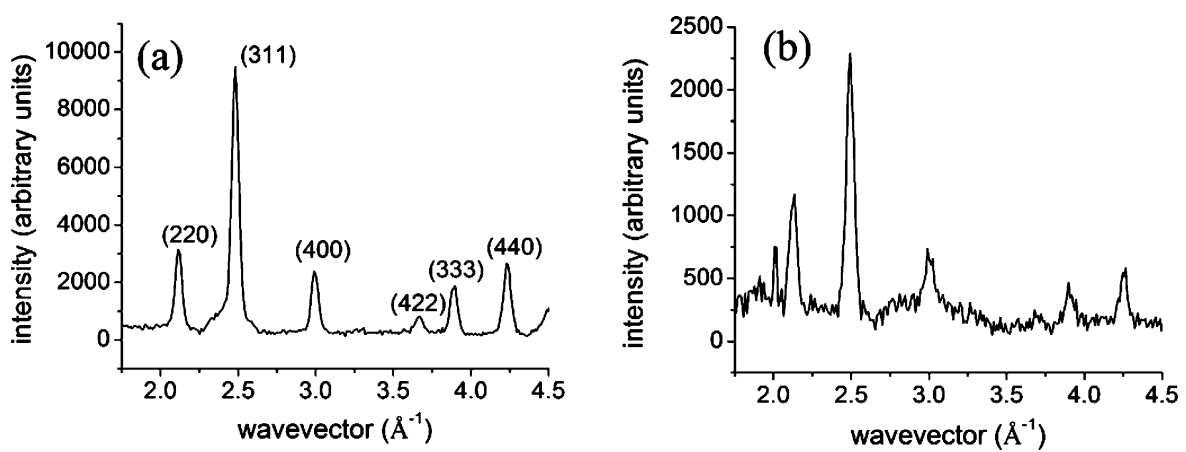

Figure 2. X-ray diffraction patterns of $12-\mathrm{nm} \mathrm{MnFe}_{2} \mathrm{O}_{4} \mathrm{NPs}$ as deposited onto glass substrates from dispersions in (a) hexane and (b) water. Intensity is plotted as a function of wave vector $((4 \pi / \lambda) \sin \theta)$. The peaks in (a) are labeled with the indexed $\mathrm{Bragg}$ reflections of the $\mathrm{MnFe}_{2} \mathrm{O}_{4}$ structure.

at $100{ }^{\circ} \mathrm{C}$. XRD data were collected using $\mathrm{Co} \mathrm{K} \alpha$ radiation $(\lambda=1.78892 \AA)$ with a Seimens D-500 diffractometer.

Magnetometry measurements were taken with a Quantum Design MPMS SQUID magnetometer. Samples were prepared by drying the NP dispersion onto a Si substrate under vacuum at $100{ }^{\circ} \mathrm{C}$.

Scanning electron microscopy (SEM) images were obtained with a Carl Zeiss Leo1560 operated at $3 \mathrm{keV}$.

Optical and epifluorescent images were obtained with a Reichert-Jung Polyvar-Met microscope. Chroma Technology Blue filter set 11001v2 was used for epifluoresence microscopy.

Magnetic scans of substrate-bound NPs were obtained using a scanning-SQUID microscope with an integrated $85.3 \mu \mathrm{m}^{2}$ pickup loop and a spatial resolution of $\sim 10 \mu \mathrm{m} .{ }^{12,13}$ This SQUID was modulated at $100 \mathrm{kHz}$ using a standard flux modulation and feedback method. Data were taken with the SQUID pickup loop $\sim 2 \mu \mathrm{m}$ from the substrate.

Magnetic-tunnel-junction (MTJ) magnetoresistance measurements were conducted as follows. The MTJ was electrically contacted with an Alessi REL-3200A probe station. Magnetoresistance was determined by supplying a $100-\mu \mathrm{A}$ current source across the junction and measuring the voltage (Agilent 4156B Parameter Analyzer) across it as an external magnetic field along the field-sensing direction was varied. All measurements were taken with a 2-cm-diameter magnetic disk mounted just beneath the MTJ substrate, creating a 220 Oe field perpendicular to the substrate. The center of the magnetoresistive effect was observed at an applied field of approximately -40 Oe. Small deviations such as this from zero field are typical of MTJs supplied by Micro Magnetics, Inc.

\section{Results and Discussion}

We chose monodisperse $\mathrm{MnFe}_{2} \mathrm{O}_{4}$ NPs as a model system for bio-functionalization, immobilization, and detection. $\mathrm{MnFe}_{2} \mathrm{O}_{4}$ has a very low magnetocrystalline anisotropy and a high moment compared with other high-moment ferrites of $\mathrm{Fe}_{2} \mathrm{O}_{3}, \mathrm{Fe}_{3} \mathrm{O}_{4}$, and $\mathrm{CoFe}_{2} \mathrm{O}_{4} \cdot{ }^{14}$ At sizes below $20 \mathrm{~nm}, \mathrm{MnFe}_{2} \mathrm{O}_{4}$ NPs are superparamagnetic at room temperature, and thus they are ideal for MTJ-based sensor detection. Our 12-nm $\mathrm{MnFe}_{2} \mathrm{O}_{4}$ NPs were synthesized following known methods ${ }^{4}$ (see Materials and Methods). As synthesized, these particles are coated with a layer of oleate and oleylamine. They are hydrophobic and can only be dispersed in a hydrocarbon solvent such as hexane.

The NPs were made water soluble following earlier work on the phase exchange of 3-nm semiconducting quantum dots. ${ }^{2}$ Having evaporated the hexane, resuspended the particles in chloroform, and added the phospholipid biotinPEG(2000)-PE (or, alternately, maleimidePEG(2000)-PE or mPEG(2000)-PE) to the dispersion, we found that after shaking for $1 \mathrm{~h}$ and
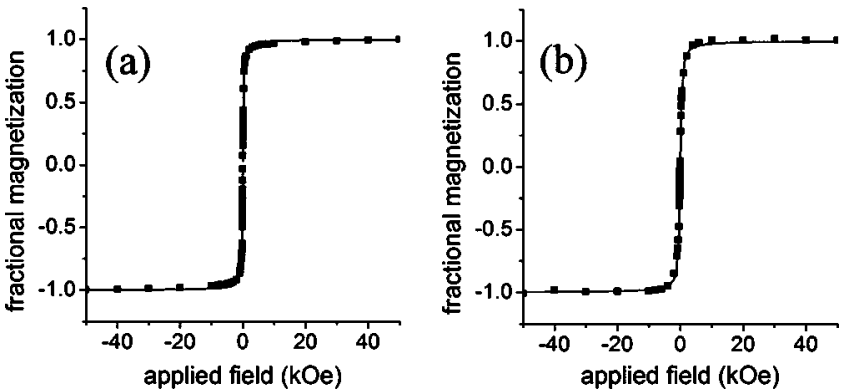

Figure 3. Magnetic moment as a function of applied magnetic field measured for 12-nm $\mathrm{MnFe}_{2} \mathrm{O}_{4} \mathrm{NPs}$ as deposited from dispersions in (a) hexane and (b) water. Data were collected at $300 \mathrm{~K}$. Solid lines are best fits of the data to the Langevin paramagnetic function (see text). Moment is plotted as a fraction of saturation moment as determined by fits to the Langevin function.

evaporating the chloroform, the particles readily dissolved in a phosphate-buffered saline (PBS) solution.

Poly(ethylene glycol) phosphatidylethanolamines (PEG-PEs) form micelles in water with inner diameters of approximately $3 \mathrm{~nm} .{ }^{15}$ Quantum dots of this diameter were chosen in earlier work $^{2}$ so that a single dot would just fill a micellar hydrophobic core. Interestingly, we find that PEG-PEs will surround and biofunctionlize particles at least as large as $15 \mathrm{~nm}$, suggesting a more direct interaction between the surface of the $\mathrm{MnFe}_{2} \mathrm{O}_{4} \mathrm{NPs}$ and the alkyl chains of the PEG-PE.

In solution, the hydrocarbon chains from the stearyl groups of the phosphatidylethanoamines (PEs) are situated at the particle surface, while the hydrophilic poly(ethylene glycol) (PEG) is in contact with the water, as illustrated in Figure 1a. Figure 1b shows a TEM image of the as-synthesized hydrophobic 12-nm $\mathrm{MnFe}_{2} \mathrm{O}_{4} \mathrm{NPs}$, and Figure 1c is an image of biotinPEG(2000)$\mathrm{PE}$ coated $\mathrm{MnFe}_{2} \mathrm{O}_{4} \mathrm{NPs}$ evaporated from water. The watersoluble sample is negatively stained with $1 \%$ phosphotungstic acid; the unstained, hydrophobic phosopholipids appear as white rings around the metallic NPs, demonstrating that it is indeed this end of the PEG-PEs which is bound to the particle surface.

Figure 2a shows X-ray diffraction (XRD) data for the nanoparticles (NPs) as synthesized. The peaks are labeled with the indexed Bragg reflections of the $\mathrm{MnFe}_{2} \mathrm{O}_{4}$ structure. The widths of the observed peaks correspond to a crystallite size of $11.4 \mathrm{~nm}$, indicating that the NPs are single crystalline. Figure $2 \mathrm{~b}$ shows comparable XRD data for NPs following functionalization with biotinPEG(2000)-PE and dispersion in water. The peak positions are unchanged while their widths have increased approximately $10 \%$, indicating that the crystal structure is substantially unchanged. The lower signal-to-noise ratio in Figure $2 b$ is due to a less dense deposition of NPs on the XRD 

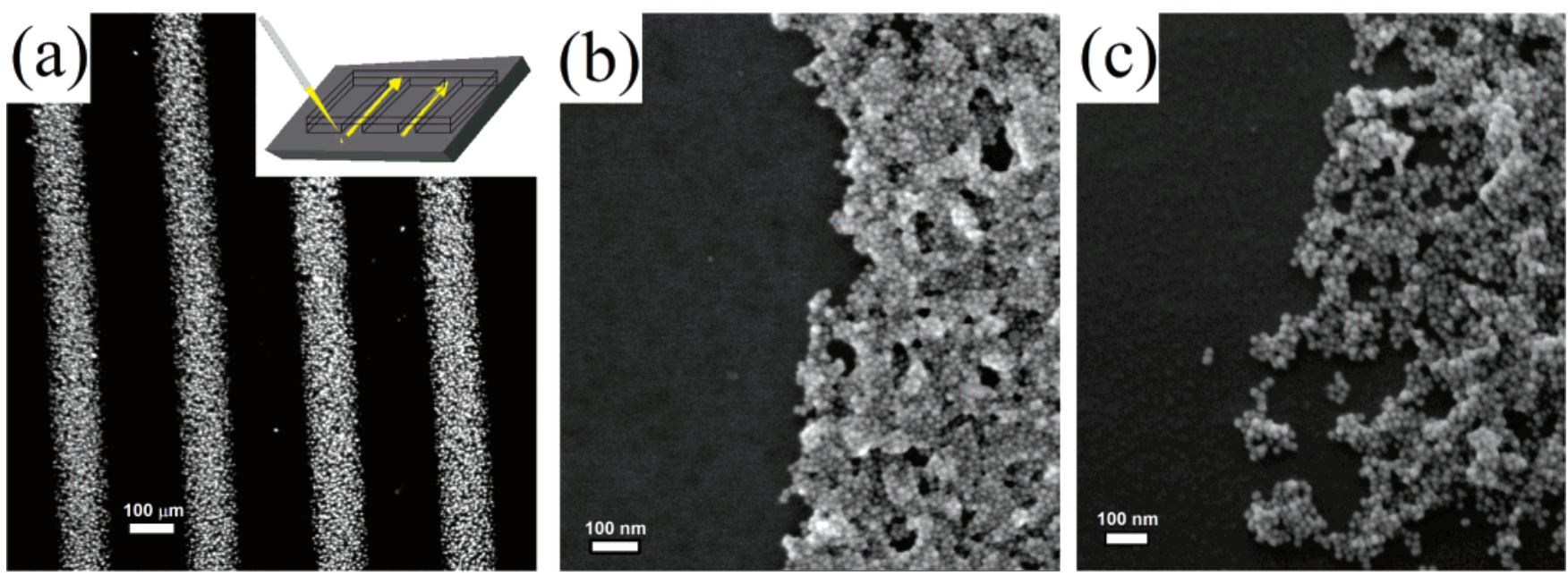

Figure 4. (a) Epifluoresence microscope image of FITC-labeled avidin patterned on a $\mathrm{SiO}_{2}$ substrate. (inset) Schematic of the patterning technique of flowing protein through microchannels of PDMS stamp on substrate. (b) SEM micrograph of biotinPEG(2000)-PE conjugated $\mathrm{MnFe}_{2} \mathrm{O}_{4} \mathrm{NPs}$ bound to the avidin-patterned region of a $\mathrm{SiO}_{2}$ substrate, as observed at a boundary of the patterning. (c) SEM micrograph of ssDNA-conjugated $\mathrm{MnFe}_{2} \mathrm{O}_{4} \mathrm{NPs}$ bound to $\mathrm{SiO}_{2}$ substrate patterned with complementary ssDNA, as observed at a boundary of the patterning.

substrate as well as the larger volume fraction occupied by the organic molecules.

Figure 3a shows the magnetization of the NPs as synthesized as a function of applied field. As expected, the particles are superparamagnetic at room temperature, and thus may be modeled with the Langevin paramagnetic function: ${ }^{16} M(x)=$ $N \mu(\operatorname{coth} x-(1 / x))$, where $x=\mu H / k_{\mathrm{B}} T, N$ is the number of NPs, $\mu$ is the magnetic moment of an individual NP, $H$ is the applied field, $k_{\mathrm{B}}$ is Boltzmann's constant, and $T$ is the absolute temperature. Fitting the data in Figure $3 a$ to this function, we find that the magnetic moment of a single particle is $3.6 \times$ $10^{-16}$ emu. Figure $3 \mathrm{~b}$ shows the magnetization of the NPs following functionalization with biotinPEG(2000)-PE and dispersion in water. The particles clearly remain superparamagnetic, although fitting the data in Figure $3 \mathrm{~b}$ to the Langevin paramagnetic function yields a somewhat reduced magnetic moment per particle of $1.9 \times 10^{-16} \mathrm{emu}$, corresponding to a magnetic radius approximately $80 \%$ that of the as-synthesized value.

Taken together, the structural data of Figures 1b,c and 2, along with the magnetometry measurements of Figure 3, demonstrate that the biofunctionalization of the NPs does not significantly alter their structure and that they remain superparamagnetic.

To demonstrate that biotin and ssDNA which we have attached to magnetic NPs remain biologically active, we sitespecifically bound the functionalized NPs to appropriately patterned substrates. ${ }^{17}$ The biotinPEG(2000)-PE-coated NPs are functionalized with biotin at the end of each PEG chain. These NPs were used for site-specific binding to avidin-patterned substrates. The particles coated with the nonfunctionalized mPEG(2000)-PE were used in control experiments. The maleimide-functionalized particles were subsequently bound to 5 'thiolated ssDNA which, in turn, was site-specifically bound to substrates bound with complementary ssDNA.

To prepare an avidin-patterned substrate, a PDMA stamp with the desired micro-fluidic network ${ }^{18}$ was prepared and placed atop an amine-functionalized ${ }^{11,19} \mathrm{SiO}_{2}$ substrate. Drops of FITClabeled avidin were then placed at one end of the microchannels, through which they flowed by capillary action (Figure 4a inset). To remove unbound protein from the substrate, the channels were flushed with $1 \%$ BSA in PBS solution. The stamp is removed from the substrate while it is immersed in 1\% BSA solution, and the substrate surface is further blocked for nonspecific binding by incubation in this solution for $10 \mathrm{~min}$ (see Materials and Methods for details). The result of this procedure is that avidin is bound to the substrate wherever microchannels had been present in the stamp, while the blocking agent BSA is bound to the rest of the substrate. The patterned FITC-labeled avidin is observable by epifluorescence microscopy; an image of a substrate patterned with avidin lines is shown in Figure 4a.

The above avidin patterned substrate was incubated in a solution of biotinylated $\mathrm{MnFe}_{2} \mathrm{O}_{4}$ particles overnight, washed with DI water, and dried with $\mathrm{N}_{2}$. An SEM image of the substrate, taken at the edge of an avidin-patterned line (Figure $4 \mathrm{~b}$ ), provides clear evidence of site-specific protein binding of the magnetic NPs. The incubation of an equivalent substrate with the nonspecifically (that is, mpeg(2000)-PE) functionalized NPs showed no site-specific binding and resulted in few bound particles scattered randomly over all regions of the substrate (not shown).

Incubating an avidin-patterned substrate overnight with a 5'-biotinylated ssDNA 20mer resulted in a ssDNA-patterned substrate (see Materials and Methods). Such a substrate was subsequently incubated overnight with $\mathrm{MnFe}_{2} \mathrm{O}_{4} \mathrm{NPs}$ functionalized with the complementary ssDNA 20mer. Following washing and drying, the sample was studied with SEM. The image in Figure 4c, taken at an edge of a ssDNA-patterned line, provides a clear demonstration that magnetic NPs can be labeled with ssDNA and site-specifically bound to a substrate patterned with complementary ssDNA. Although our magnetic detection measurements have been carried out exclusively on biotinylated NPs bound to an avidin-patterned substrate, Figure 4b,c indicates that DNA-fuctionalized particles could be detected just as effectively.

An image of the magnetic field above a substrate to which magnetic NPs had been site-specifically bound was obtained using a scanning-SQUID microscope. A topograph of the flux measured across a substrate is shown in Figure 5a; the two regions containing magnetic NPs (at the left and right of the image) exhibit significant magnetic fluctuations. These are separated by a NP-free region which exhibits relatively few such fluctuations. As these data were collected at $4 \mathrm{~K}$, following cooling of the sample in a negligible magnetic field, the moment of each NP is frozen in a random direction coincident with one of its magneto-anisotropic axes. Thus, the average magnetic flux 

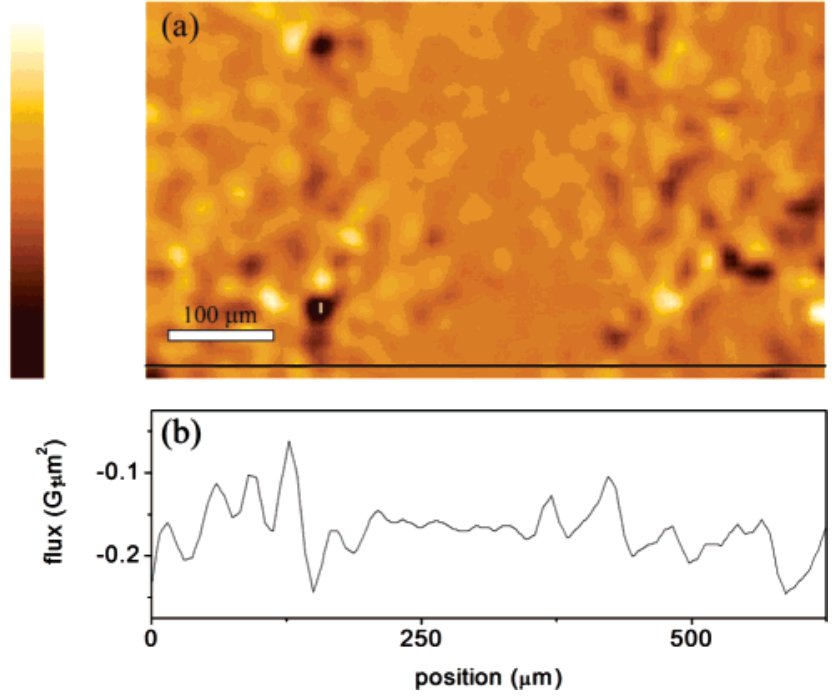

Figure 5. (a) SQUID microscope image at $4 \mathrm{~K}$ of magnetic flux above a $\mathrm{SiO}_{2}$ substrate which has biotinPEG(2000)-PE-conjugated $\mathrm{MnFe}_{2} \mathrm{O}_{4}$ NPs bound to avidin-patterned regions (the right and left sides of the image). Fluctuations in flux above NP-bound regions result from fluctuations in the average of the randomly oriented, frozen particle moments (see text). (b) Plot of magnetic flux across black line in (a).

from all of the particles should be approximately zero. As the SQUID microscope samples the collective moment of a finite number of NPs, one expects to measure deviations from zero which scale as the square root of the number of particles sampled. Figure $5 \mathrm{~b}$ shows a line scan of the magnetic flux through the black line in Figure 5a. As expected, the regions of the surface covered by bound NPs show magnetic fluctuations both above and below the relatively constant value observed in the region with no particles.

For the magnetic detection of NPs to be of practical utility, it is necessary that the detector be incorporated into the substrate on which the particles are bound and that the particles be detectable at room temperature. To demonstrate the feasibility of such detection, we patterned FITC-avidin on an $\mathrm{SiO}_{2}$-coated MTJ field sensor (Micro Magnetics, Inc.). The tunnel junction is comprised of a pinned magnetic layer separated from an unpinned magnetic layer by an $\mathrm{Al}_{2} \mathrm{O}_{3}$ tunneling barrier. The magnetoresistance of the junction depends on the orientation of the unpinned magnetic layer, which thus serves as a field detector. (See ref 5 for a schematic illustration of such a detector.) Figure $6 \mathrm{a}$ is an optical micrograph of the MTJ field sensor superposed with an epifluorescence image of the patterned avidin. The MTJ is located beneath the visible electrical contact at the site identified by a red ellipse. The field-sensing direction is along the minor axis of the ellipse, and avidin was patterned to be on one side of the MTJ along this axis. A magnetic disk with a 2-cm diameter was placed beneath the MTJ substrate, creating a 220-Oe field perpendicular to the substrate. This field has the effect of partially aligning the moments of any bound NPs without affecting the performance of the MTJ.

Prior to binding any NPs to the substrate, the change in resistance across the MTJ, as a function of a magnetic field applied along the field-sensing direction, is plotted as the red line in Figure 6b. The black line shows an equivalent scan following incubation of the avidin-patterned MTJ substrate with biotin-functionalized $\mathrm{MnFe}_{2} \mathrm{O}_{4}$ NPs. When a field is applied along the sensing axis, the net magnetization of any bound NPs is rotated toward the sensing field axis. Because the NPs are located beside the MTJ (on one side of its field-sensing axis),
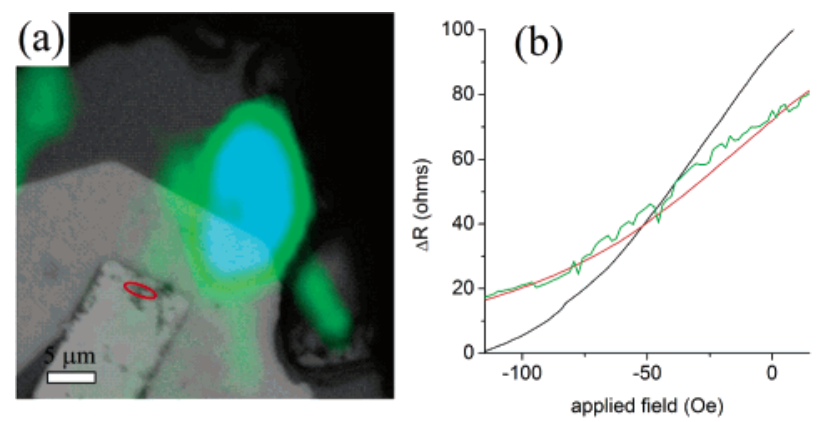

Figure 6. (a) optical micrograph of magnetic-tunnel-junction field sensor superposed with epifluorescence image of FITC-labeled avidin which has been patterned onto the surface of the sensor substrate. The location of the actual tunnel junction (below the visible contact) is shown with a red ellipse. (b) Magnetoresitance of MTJ as a function of magnetic field applied along the field-sensing direction. Red and black lines correspond to measurements taken before and following site-specific binding of magnetic NPs, respectively. Green line corresponds to data taken following removal of NPs. A constant resistance (that is, not due to magnetoresistive effect) has been subtracted from each of the data lines.

the dipole field generated by these magnetized NPs will enhance the field observed by the MTJ sensing layer and, as a result, the slope of the magnetoresistance as a function of applied field will increase. This is precisely what we observe. To verify that the change in magnetoresistance was not a consequence of removing the sample from the experimental probe station and then remounting it following NP binding, we removed the NPs and avidin patterning by wetting the substrate with acetone and gently rubbing it with a cotton swab while it remained fixed in the probe station. Following removal of the magnetic NPs, the measured magnetoresistance is shown as the green line in Figure $6 \mathrm{~b}$. The slope has returned to its pre-NP hybridization value, although the signal is now noisier, presumably because traces of acetone are still present at the probe electrodes.

\section{Conclusions}

This report demonstrates that monodisperse magnetic NPs can be successfully bio-functionalized with either proteinbinding molecules or ssDNA, that the molecules on the surface of these particles remain active and can be bound to protein or complementary ssDNA-patterned substrates, and that these bound particles can be magnetically detected in situ at room temperature with a MTJ sensor. The successful bio-functionalization of the particles allows for the potential introduction of these particles to both in-vitro and in-vivo biological environments. The magnetic detection of these particles demonstrates the viability of utilizing magnetic NPs to detect both proteins and DNA.

Acknowledgment. We thank L. Huang, R. Sandstrom, C. Black, J. Rosner, and N. Ruiz for technical assistance, B. Michel, R. Koch, and D. Abraham for helpful discussions, and G. Grinstein for a careful reading of the manuscript. This work was supported in part by DARPA/ONR under Grant No. N00014-01-1-0885 and also in part by the MRSEC Program of the NSF at Columbia Univerisity under Award Number DMR-0213574.

\section{References and Notes}

(1) Li, G. X.; Wang, S. X. IEEE Trans. Magn. 2003, 39, 3313.

(2) Dubertret, B.; Skourides, P.; Norris, D. J.; Noireaux, V.; Brivanlou, A. H.; Libchaber, A. Science 2002, 298, 1759.

(3) Hyeon, T. Chem. Commun. 2003, 927. 
(4) Sun, S. H.; Zeng, H.; Robinson, D. B.; Raoux, S.; Rice, P. M.; Wang, S. X.; Li, G. X. J. Am. Chem. Soc. 2004, 126, 273.

(5) Liu, X. Y.; Ren, C.; Xiao, G. J. Appl. Phys. 2002, 92, 4722.

(6) Li, G. X.; Joshi, V.; White, R. L.; Wang, S. X.; Kemp, J. T.; Webb, C.; Davis, R. W.; Sun, S. H. J. Appl. Phys. 2003, 93, 7557.

(7) Edelstein, R. L.; Tamanaha, C. R.; Sheehan, P. E.; Miller, M. M.; Baselt, D. R.; Whitman, L. J.; Colton, R. J. Biosens. Bioelectron. 2000, 14 , 805.

(8) Kim, H. J.; Jang, S. H.; Oh, K. H.; Kim, T. S.; Kim, K. Y. Phys. Status Solidi A-Appl. Res. 2004, 201, 1961.

(9) Black, C. T.; Murray, C. B.; Sandstrom, R. L.; Sun, S. H. Science 2000, 290, 1131.

(10) Park, S. J.; Taton, T. A.; Mirkin, C. A. Science 2002, 295, 1503.

(11) Ahmed, A.; Bonner, C.; Desai, T. A. Biomed. Microdevices 2001, 3,89 .
(12) Kirtley, J. R.; Tsuei, C. C.; Moler, K. A.; Kogan, V. G.; Clem, J. R.; Turberfield, A. J. Appl. Phys. Lett. 1999, 74, 4011.

(13) Kirtley, J. R.; Ketchen, M. B.; Stawiasz, K. G.; Sun, J. Z.; Gallagher, W. J.; Blanton, S. H.; Wind, S. J. Appl. Phys. Lett. 1995, 66, 1138.

(14) West, A. R. Basic Solid State Chemistry; Wiley: New York, 1988. (15) Johnsson, M.; Hansson, P.; Edwards, K. J. Phys. Chem. B 2001, 105,8420

(16) Bean, C. P.; Livingston, J. D. J. Appl. Phys. 1959, 30, 120 S.

(17) Mann, S.; Shenton, W.; Li, M.; Connolly, S.; Fitzmaurice, D. Adv. Mater. 2000, 12, 147.

(18) Delamarche, E.; Bernard, A.; Schmid, H.; Bietsch, A.; Michel, B.; Biebuyck, H. J. Am. Chem. Soc. 1998, 120, 500.

(19) Nashat, A. H.; Moronne, M.; Ferrari, M. Biotechnol. Bioeng. 1998, 60,137 